\title{
ASOCIACIÓN ENTRE CONCENTRACIONES DE FERRITINA, CRP, sTFR Y HbA1c EN SUJETOS CON Y SIN DM2.
}

\author{
ASSOCIATION BETWEEN CONCENTRATIONS OF FERRITIN, CRP, STFR AND HbA1C IN SUBJECTS WITH \\ AND WITHOUT DM2.
}

Márquez Ibarra Adriana Alejandra ${ }^{1}$, González Ponce Ana María².

1 Universidad Tecmilenio, Zapopan, Jalisco, México. 2 Universidad del Papaloapan, Tuxtepec, Oaxaca, México.

Citation: Márquez Ibarra A.A., González Ponce A.M., (2020) Asociación entre concentraciones de ferritina, CRP, sTFR y HbA1c en sujetos con y sin DM2. Revista Salud Pública y Nutrición, 19 (3), 8-18.

Editor: Esteban G. Ramos Peña, Dr. CS., Universidad Autónoma de Nuevo León, Facultad de Salud Pública y Nutrición, Monterrey Nuevo León, México. Copyright: (C2020 Márquez Ibarra A.A., et al. This is an open-access article distributed under the terms of Creative Commons Attribution License [CC BY 4.0], which permits unrestricted use, distribution, and reproduction in any medium, provided the original author and source are credited.

Competing interests: The authors have declared that no competing interests exist.

DOI: https://doi.org/10.29105/respyn19.3-2

Recibido: 11 de mayo 2020;

Aceptado: 10 de agosto 2020

Email: aami27@hotmail.com 


\title{
ASOCIACIÓN ENTRE CONCENTRACIONES DE FERRITINA, CRP, STFR Y HbA1C EN SUJETOS CON Y SIN DM2.
}

\author{
Márquez Ibarra Adriana Alejandra1, González Ponce Ana María². \\ 1 Universidad Tecmilenio, Zapopan, Jalisco, México. 2 Universidad del Papaloapan, Tuxtepec, Oaxaca, México.
}

\begin{abstract}
RESUMEN
Introducción. Se ha demostrado que existe un componente inflamatorio en la patogenia de la diabetes tipo 2 (DM2) y se ha asociado con elevaciones en los niveles de ferritina sérica. Objetivo: Evaluar la asociación entre concentraciones séricas de ferritina, CRP, receptores solubles de transferrina (sTfR) y hemoglobina glucosilada (HbA1c) en sujetos con y sin diabetes tipo 2. Material y Método: Estudio secundario, derivado de la ENSANUT 2006. Se analizaron concentraciones de ferritina, sTFR y CRP $(n=4578)$, se asoció HbA1c y ferritina $(n=287)$, y variables como, índice de masa corporal (IMC), edad, sexo, tratamiento actual para DM2, tabaquismo y consumo de alcohol. El análisis estadístico se realizó con modelos de regresión logística múltiple y modelos de regresión lineal múltiple. Se consideró como significancia estadística un valor de $p<0.05$ y la dirección y magnitud de la asociación. Resultados: La posibilidad de tener DM2 se asoció con las concentraciones séricas de ferritina y con las de CRP $(p<0.02)$. La ferritina se asoció de manera inversa con las de sTFR ( $\beta-0.91, p<0.001)$. La HbA1c no se asoció con la ferritina. Conclusiones: En mexicanos con DM2 la ferritina sérica es mayor que en sujetos con diabetes y su variabilidad está explicada por indicadores del estatus de hierro y de inflamación.

Palabras Clave: Diabetes tipo 2, inflamación, proteína c- reactiva, ferritina.
\end{abstract}

\section{ABSTRACT}

Introduction: An inflammatory component has been shown to exist in the pathogenesis of type 2 diabetes (DM2) and has been associated with elevations in serum ferritin levels. Objective: to evaluate the association between serum ferritin concentrations, CRP, soluble transferrin receptors (sTfR) and glycosylated hemoglobin (HbA1c) in subjects with and without type 2 diabetes. Material and method: Secondary study, derived from ENSANUT 2006. Concentrations of ferritin, sTFR and CRP ( $n=4578)$ were analyzed, HbA1c and ferritin $(n=287)$ were associated, and variables such as, body mass index (BMI), age, sex, current treatment for DM2, smoking and alcohol consumption. Statistical analysis was performed with multiple logistic regression models and multiple linear regression models. An association with a value of $p<0.05$ was considered significant and the direction and magnitude of the association. Results: The possibility of having DM2 was associated with serum ferritin and CRP concentrations $(p<0.02)$. Ferritin was inversely associated with sTFR $(\beta-0.91, p<0.001)$. HbA1c was not associated with ferritin. Conclusions: In Mexicans with DM2 serum ferritin is higher than in subjects with diabetes and its variability is explained by indicators of iron status and inflammation.

Key words: Type 2 diabetes, inflammation, c-reactive protein, ferritin. 


\section{Introducción}

La diabetes mellitus tipo 2 (DM2) es una causa importante de morbimortalidad en el mundo. En México la Encuesta Nacional de Salud y Nutrición de medio camino (ENSANUT-MC) (2016), encontró que la prevalencia de diabetes en el país pasó de $9.2 \%$ en 2012 a 9.4\% en 2016, esto con base a diagnóstico previo de la enfermedad. Las mujeres reportan mayores valores $(10.3 \%)$ que los hombres $(8.4 \%)$. Esta tendencia se observa tanto en localidades urbanas como en rurales $(9.5 \%$ en mujeres, $8.9 \%$ en hombres) (Rojas et al., 2018).

La DM2 tiene una etiología multifactorial incluyendo factores ambientales (dietéticos, obesidad, tabaquismo, alcoholismo, sedentarismo, etc.) y genéticos. Desde 1998 se ha documentado evidencia epidemiológica de la asociación entre la ferritina y DM2, en donde se ha evaluado la reserva de hierro corporal como predictor de DM2, encontrando que los hombres con concentraciones elevadas de ferritina tienen más posibilidad de tener DM2 en comparación con los hombres con bajos niveles de ferritina, además se ha observado que la ferritina sérica se asocia positivamente con las concentraciones de insulina, hemoglobina glucosilada (HbA1c) y glucosa sérica en ayuno; siendo más significativa en hombres. Los resultados también han evidenciado que las personas con DM2 tienen una media de ferritina sérica mayor al compararlos con grupos control y que tanto ferritina como transferrina se asocian de manera independiente con la aparición de hiperglucemia (Canturk et al.,2003; Ford y Cogswell, 1999; Forouhi et al., 2007; Fumeron, P'eanc, Pharmd y Balkau., 2006; Jiang,2004; Salonen et al.,1998; Sharifi y Sazandeh,2004). Sin embargo, los resultados de la asociación entre la concentración de ferritina sérica y el riesgo de diabetes tipo 2 en diversos tipos de estudios, han sido inconsistentes. Varios de estos han observado una asociación positiva; sin embargo, las concentraciones de ferritina en el suero también pueden reflejar una inflamación sistémica que coexiste con la diabetes, más que concentraciones altas de hierro, ya que las muestras de sangre se recogen después del diagnóstico de diabetes. Jiang et al., (2004) realizaron un estudio prospectivo de casos y controles anidado dentro de la cohorte de enfermeras, de las 32,826 mujeres que proporcionaron muestras de sangre durante 19891990 y estaban libres de diabetes diagnosticada, enfermedad cardiovascular y cáncer, 698 desarrollaron diabetes durante los 10 años de seguimiento. Los resultados señalaron que la ferritina fue mayor (109 [105] contra 71.5 [68.7] $\mathrm{ng} / \mathrm{mL}$ en los controles; $\mathrm{p}<0.001)$ y la relación de los sTfR era más baja (102 [205] contra 141 [340], respectivamente; $\mathrm{p}<=0.01)$. Los RR a través de los quintiles de los sTfR fue de 2.44 (IC 95\%, 1.613.71), 1.00 (IC 95\%, 0.64-1.56), 1.13 (IC 95\%, 0.73 1.74), 0.99 (IC 95\%, 0.64-1.53) y $1.00(\mathrm{p}=0.01)$, por lo tanto, la ferritina elevada y un cociente bajo de sTfR, se puede asociar a un progresivo riesgo de DM2 en mujeres sanas.

Jehn et al., (2007) en una cohorte anidada dentro del estudio de riesgo de aterosclerosis en las comunidades (ARIC), y con el objetivo de determinar la asociación entre el nivel de ferritina en plasma y el riesgo de DM2, encontraron que después de ajustar por edad, sexo, estado menopáusico, etnia, centro, tabaquismo e ingesta de alcohol, el RR para DM2, comparando el quinto quintil de ferritina con el primer quintil, fue 1.74 (IC 95\%: 1.14- 2.65; p $<0,001)$. Después de un ajuste adicional para el IMC y los componentes del síndrome metabólico, el RR fue de 0.81 (IC 95\%: 0.49- 1.34; p= 0.87). En un estudio anidado de casos y controles, los casos tuvieron niveles de sTfR más altos $(3.50 \pm 0.07$ vs $3.30 \pm 0.06 \mathrm{mg} / 1 ; \mathrm{p}=0.03$ ), pero los niveles de ferritina no fueron estadísticamente diferentes. El OR y el IC 95\% para la incidencia de DM2 que comparó los cuartiles más altos con los más bajos de sTfR fue 2.26 (1.37-4.01; $\mathrm{p}=0.008)$. Los autores concluyeron que los niveles de sTfR moderadamente elevados se asocian con un mayor riesgo de DM2 entre las personas con sobrepeso y obesidad (Rajpathak,2009). Sun, et al., (2013) en un estudio prospectivo cuyo objetivo fue investigar prospectivamente la asociación ferritina-diabetes, se midió ferritina plasmática, CRP, adiponectina y glutamil transferasa (GGT), después de múltiples ajustes, la asociación fue significativa con un RR de DM2 de 1.90 (IC 95\%: 1.37-2.65) al comparar el quintil más alto con el más bajo de ferritina específico por sexo. Además, en este mismo estudio, se incluyeron 9 estudios adicionales. El RR agrupado fue 1,60 (IC 95\%: 1.25- 2.04) al comparar la categoría más alta con la más baja de ferritina con una heterogeneidad moderada (I $(2)=49.0 \%$; $\mathrm{p}=$ 0.03 ), en este metaanálisis se detectó una relación lineal significativa dosis-respuesta. 
La plausibilidad biológica hasta ahora propuesta pone de manifiesto que el metabolismo de la insulina se afecta con el aumento de las reservas de hierro, lo que conduce a la hiperinsulinemia periférica. De hecho, la anormalidad inicial y más común observada en condiciones de sobrecarga de hierro es la resistencia a la insulina en el hígado, así mismo el estrés oxidativo induce resistencia a la insulina, al disminuir su internalización y al aumentar la síntesis de ferritina. (Fernández-Real, López-Bermejo y Ricart, 2002). La ferritina actúa como mediador inflamatorio al activar la proteína quinasa activadora de mitogénesis (MAPK) y el factor nuclear kappabeta $(\mathrm{FN}-\mathrm{K} \beta)$, que junto con una serie de reacciones con especies reactivas de oxígeno (ROS) activan la muerte celular programada y la respuesta inflamatoria (Carrillo, Peña y Zepeda, 2015).

Ma (2018) en su trabajo concluyo que los niveles elevados de ferritina sérica se asociaron con medidas sustitutivas de la resistencia a la insulina entre los hombres de mediana edad y ancianos y las mujeres posmenopáusicas, pero no en las mujeres premenopáusicas.

En la revisión realizada por Swaminathan et al. (2002), se concluyó que la sobrecarga de hierro no es un requisito previo para que intervenga en la diabetes y sus complicaciones.

Así mismo, Eshed, Elis y Lishner (2001) en una revisión crítica sobre la asociación entre DM2, resistencia a la insulina, control glucémico, complicaciones diabéticas e hiperferritinemia, encontró que la concentración de ferritina en plasma se correlaciona positivamente con la resistencia a la insulina y con el riesgo de presentar DM2, pero la sobrecarga de hierro sustancial no es una característica típica de la DM2, y que por lo tanto no hay correlación entre el nivel de ferritina en plasma y el control glucémico o las complicaciones microangiopáticas diabéticas.

En otra revisión realizada por Pickup (2004), se encontraron resultados contradictorios, y concluye que se necesita más investigación para confirmar y aclarar en qué medida la inflamación en la diabetes tipo 2 es una anormalidad primaria o parcialmente secundaria a hiperglucemia, obesidad, aterosclerosis u otras características comunes de la enfermedad.
El significado de esta asociación no está claramente definido por lo cual, el presente estudio tuvo como objetivo evaluar la asociación entre concentraciones séricas de ferritina, CRP, receptores solubles de transferrina (ya que es necesario saber si la mayor variabilidad de su concentración de debe per se al estatus de hierro) y hemoglobina glucosilada (HbA1c) en sujetos con y sin diabetes tipo 2 .

\section{Material y Método}

Diseño y población de estudio:

El presente es un estudio transversal, que constituye un análisis secundario de la base de datos de adultos $>20$ años, de ambos sexos, de la Encuesta Nacional de Salud y Nutrición (ENSANUT,2006). Esta encuesta tuvo un diseño muestral probabilístico, polietápico, estratificado y por conglomerados, para mayores detalles se puede consultar la metodología de esta (ENSANUT,2006). El protocolo fue aprobado por las comisiones de Ética, Investigación y Bioseguridad del Instituto Nacional de Salud Pública (INSP).

Los datos utilizados en el estudio se derivaron de la submuestra de 6350 sueros seleccionados al azar de 12,633 muestras de suero disponibles, los análisis se realizan de forma individual.

El cálculo del tamaño de la submuestra general de la ENSANUT 2006 se basó en los siguientes supuestos: detectar una prevalencia del $8.2 \%$ de diabetes, con un nivel de confianza del $95 \%$, con una tasa de no respuesta del $20 \%$ y un efecto de diseño de 1.7 (basado en estimaciones de la Encuesta Nacional de Nutrición 1999 y la Encuesta Nacional de Salud 2000), la submuestra se calculó originalmente para que fuera representativa a nivel nacional y de las cuatro regiones geográficas de México, es decir Norte, Centro, Centro-Oeste, Sur-Sureste.

Para este estudio se consideró la submuestra original de la ENSANUT 2006 y se incluyeron los sujetos que tuvieran disponibles todas las variables de interés: concentraciones de ferritina, sTFR, CRP $(n=4578)$ independientemente de la presencia o ausencia de DM2, es decir, se consideraron todos los adultos $\geq 20$ años con las variables de interés en la base de datos; se realizó como ejercicio el cálculo de muestra para verificar cumplir con datos suficientes, para el primer análisis (asociación ferritina y la presencia de DM2) se llevó a cabo el cálculo de tamaño de muestra para 

ferritina.

diferencias de medias para grupos con y sin diabetes, estratificando por sexo, tomando como referencia la investigación (Forouhi et al.,2007) y considerando el efecto de diseño (DEF) de la ENSANUT, estimando un efecto pequeño y usando el comando sampsi del programa Stata, se obtuvo como máximo una $\mathrm{n}=2125$ y para el segundo y tercer análisis se realizó cálculo del poder estadístico para regresión lineal, considerando varios escenarios con distintas $\mathrm{r} 2, \mathrm{y}$ utilizando el comando powerreg, obteniendo como máximo una n de 800; por lo tanto fue viable tomar los 4,578 datos disponibles como muestra.

Para el análisis de la asociación entre hemoglobina glucosilada (HbA1c) y las concentraciones séricas de ferritina, la muestra es no probabilística, limitándose a solo 287 sujetos de los 1,099 que tenían un diagnóstico previo de DM2 hecho por un médico contaban con determinaciones simultaneas de HbA1c y ferritina (Villalpando, 2010).

Se excluyeron del análisis a sujetos con niveles de ferritina $\geq 1000 \mathrm{ng} / \mathrm{ml}$, aquellos que auto reportaron transfusiones o donaciones de sangre en el último año, mujeres embarazadas y en estado de lactancia, los criterios de no inclusión fueron datos insuficientes de todas las variables de interés en la base de datos o que presentaran errores en su captura.

Medición y definición de las variables.

Variables socioeconómicas y clínicas:

Para fines de esta investigación se utilizaron datos obtenidos de los cuestionarios sociodemográfico y de salud de adultos > 20 años, aplicado en la ENSANUT (Shamah-Levy, Villalpando-Hernández y RiveraDommarco, 2007).

Las variables extraídas de estos cuestionarios fueron edad (años), sexo, tratamiento actual para la DM2 (insulina, hipoglucemiantes, ambos o ninguno), tabaquismo, definido como haber fumado por lo menos 100 cigarros en el último año y estratificado, no, si o nunca y además el consumo de alcohol actual, clasificado como nunca, diario, semanal, mensual u ocasional.

Antropometría:

Para la medición del peso corporal se utilizaron balanzas electrónicas marca Tanita con una precisión de 100 g, su funcionamiento y calibración se verificó con la ayuda de taras u objetos con peso conocido. Para la medición de la talla se utilizó un estadímetro marca (Dynatop, México, D.F.) con capacidad de 2 $\mathrm{m}$ y una precisión de $1 \mathrm{~mm}$. Los encuestadores fueron previamente estandarizados y capacitados para las mediciones de acuerdo con las técnicas descritas en el manual de procedimientos para proyectos de nutrición del Instituto Nacional de Salud Pública (INSP) (Shamah-Levy, VillalpandoHernández y Rivera-Dommarco, 2006). El índice de masa corporal (IMC) fue calculado a partir del peso y la talla: peso $(\mathrm{kg}) /$ talla (m2) (OMS,2000).

Variables bioquímicas:

Para medir las variables bioquímicas se tomaron muestras sanguíneas (Twetz,1999) de manera aleatorizada en $30 \%$ de los 45,446 sujetos mayores de 20 años incluidos en la encuesta. Los sujetos fueron citados a la toma de muestras en condiciones de ayuno; en todos los casos se registró el tiempo de la última comida y el $91.3 \%$ declararon haber tenido más de 8 horas de ayuno.

Las concentraciones de HbA1c se determinaron por método inmuno colorimétrico en sangre total en un autoanalizador marca PRESTIGE 24i (Niigata, Japón), con un intervalo de medición de 2.0-16.0 \% y un coeficiente de variación (CV) del 5\%. Las determinaciones de sTFR y ferritina sérica se realizaron por el método de ELISA (Behring, Maburg, Alemania), usando un autoanalizador BN100 con un intervalo de medición para ferritina de 2$640 \mathrm{ng} / \mathrm{mL}$ y para sTFR de $0.14-8.80 \mathrm{mg} / \mathrm{L}$, con un CV para sTFR del $5 \%$ y $1.54 \%$ para ferritina. La CRP se determinó por nefelometría, usando anticuerpos monoclonales de alta sensibilidad en un nefelómetro marca (Behring, Maburg, Alemania), con un intervalo de medición de $0.17-220 \mathrm{mg} / \mathrm{L}$ y un CV del $5.29 \%$. Las concentraciones de glucosa se midieron en suero usando el método automatizado de glucosa oxidasa en un autoanalizador marca PRESTIGE 24i, (Niigata, Japón) con un CV del 5\%. Los niveles de hierro sérico se midieron en un espectrómetro de emisión óptica de plasma por acoplamiento inductivo (ICP-OES) marca Vista PRO-CCD- simultaneous (Mulgrave, Victoria, Australia) con un rango de medición de 10-400 $\mu \mathrm{g} / \mathrm{L}$ y un $\mathrm{CV}$ del $5 \%$ (Twetz,1999). 
Definición de Diabetes:

1.-Sujetos que declararan haber tenido un diagnóstico previo de DM2 establecido por un médico los cuales fueron denominados "diagnóstico previo".

2.- Sujetos cuya concentración de glucosa en la muestra de sangre en ayunas fuera $\geq 126 \mathrm{mg} / \mathrm{dL}$, denominados como "hallazgo de la encuesta".

Análisis estadístico:

Se realizó un análisis descriptivo de las variables, usando medias e intervalos de confianza (IC) al 95\%, para las variables continuas y distribución de frecuencias para las variables categóricas. Se valoraron los supuestos de normalidad, homocedasticidad, colinealidad y bondad de ajuste. La ferritina, CRP, hierro sérico y sTFR no se distribuyeron normalmente según la prueba Shapiro Will, por lo cual se realizó una transformación logarítmica, expresando los resultados post análisis como antilogarítmos. Se consideró como significativa una asociación con un valor de $\mathrm{p}<0.05$ y los IC de cada OR.

Es importante señalar que este estudio indaga sobre varios puntos a considerar por lo tanto se construyen varios modelos en los cuales las variables dependientes e independientes difieren.

1.-Para evaluar la asociación entre DM2 y ferritina se construyeron modelos de regresión logística múltiple, considerando como variable dependiente la ausencia o presencia de DM2 y como variable independiente la distribución en quintiles de ferritina, los modelos se ajustaron por CRP, IMC, edad, sexo, consumo de alcohol y tabaco.

2.-Para evaluar las asociaciones entre ferritina y CRP $y$, entre ferritina y sTFR (en el entendido que sTFR es la única variable disponible sobre el estado de hierro para el análisis y esto corroboraría que los cambios en ferritina se ven reflejados en los receptores) se construyeron modelos de regresión lineal múltiple, introduciendo como variable dependiente el logaritmo de ferritina como escala continua y como variables independientes alternativamente el logaritmo de CRP o el logaritmo de sTFR como variable continua, los modelos se ajustaron por sexo, edad, IMC, DM2, consumo de alcohol y tabaco. Se estimaron los coeficientes estandarizados para valorar la aportación de cada variable independiente sobre la variable dependiente.
3.-Para estimar la asociación entre ferritina y $\mathrm{HbA1c}$ se realizaron modelos de regresión lineal múltiple, considerando como variable dependiente el logaritmo de ferritina como variable continua y como variable independiente las concentraciones de HbA1c como variable continua, los modelos se ajustaron por IMC, edad, sexo, consumo de alcohol y tabaco, sTFR, CRP, hierro, tratamiento médico DM2.

Fue considerada como significativa una asociación con un valor de $\mathrm{p}<0.05$ para los efectos principales. Para el análisis estadístico se utilizó el programa estadístico Stata considerando el ajuste del diseño de la ENSANUT, utilizando el módulo svy con excepción del análisis de asociación entre ferritina y Hb1Ac.

\section{Resultados}

1.-Comparaciones bivariadas

Las características de la población de estudio se presentan en la tabla 1, según las pruebas t student y $\mathrm{X} 2$. La media geométrica de las concentraciones de ferritina $(80.1 \mathrm{ng} / \mathrm{mL}$ vs. $49.6 \mathrm{ng} / \mathrm{mL} \mathrm{p}<0.0001)$ y de CRP (3.34 mg/L vs $2.03 \mathrm{mg} / \mathrm{L} \mathrm{p}<0.0001)$ fueron significativamente mayores en el grupo con DM2 en comparación con el grupo sin diabetes, respectivamente; mientras que las medias de las concentraciones de sTFR no fueron significativamente diferentes entre grupos.

La media del IMC fue significativamente mayor en el grupo con DM2 $(28.77 \mathrm{~kg} / \mathrm{m} 2)$ en comparación al grupo $\sin$ DM2 $(27.54 \mathrm{~kg} / \mathrm{m} 2 \mathrm{p}<0.002)$. 
Diabetes tipo 2, inflamación, proteína c- reactiva, ferritina.

\begin{tabular}{|c|c|c|c|c|}
\hline & \multicolumn{2}{|c|}{$\begin{array}{l}\text { Diabeticos tipo } 2 \\
(n=397)\end{array}$} & \multicolumn{2}{|c|}{$\begin{array}{l}\text { No diabéticos } \\
(n=4181)\end{array}$} \\
\hline & $\mathbf{N}$ & $\%$ & $\mathrm{n}$ & $\%$ \\
\hline \multicolumn{5}{|l|}{ Edad (años) } \\
\hline $20-40 * * *$ & 52 & 13 & 2300 & 55 \\
\hline $41-60 * * *$ & 190 & 48 & 1296 & 31 \\
\hline $61-90 * * *$ & 155 & 39 & 585 & 14 \\
\hline \multicolumn{5}{|l|}{ Sexo } \\
\hline Hombre & 179 & 45 & 1923 & 46 \\
\hline Mujer & 218 & 55 & 2258 & 54 \\
\hline \multicolumn{5}{|l|}{ Tabaquismo $^{+}$} \\
\hline No & 44 & 11 & 543 & 13 \\
\hline Si** & 151 & 38 & 1129 & 27 \\
\hline Nunca* & 202 & 51 & 2509 & 60 \\
\hline \multicolumn{5}{|l|}{$\begin{array}{l}\text { Consumo de } \\
\text { alcohol** }\end{array}$} \\
\hline Nunca & 186 & 47 & 2132 & 51 \\
\hline Diario* & 20 & 5 & 84 & 2 \\
\hline Semanal & 44 & 11 & 460 & 11 \\
\hline Mensual & 12 & 3 & 209 & 5 \\
\hline \multirow[t]{2}{*}{ Ocasional } & 135 & 34 & 1296 & 31 \\
\hline & & $\begin{array}{l}\text { Media } \\
\text { geométrica } \\
\text { IC (95\%) }\end{array}$ & & $\begin{array}{l}\text { Media } \\
\text { geométrica } \\
\text { IC (95\%) }\end{array}$ \\
\hline $\mathrm{CRP}(\mathrm{mg} / \mathrm{L})^{* * *}$ & 397 & $\begin{array}{c}3.34 \\
(2.86-3.90)\end{array}$ & 4181 & $\begin{array}{c}2.03 \\
(1.93-2.14)\end{array}$ \\
\hline $\begin{array}{l}\text { Ferritina } \\
(\mathrm{ng} / \mathrm{ml})^{* * *}\end{array}$ & 397 & $\begin{array}{c}80.08 \\
(68.75-93.29)\end{array}$ & 4181 & $\begin{array}{c}49.58 \\
(46.40-52.99)\end{array}$ \\
\hline $\operatorname{sTFR}(\mathrm{mg} / \mathrm{L})$ & 397 & $\begin{array}{c}2.89 \\
(2.74-3.06)\end{array}$ & 4181 & $\begin{array}{c}2.88 \\
(2.83-2.93)\end{array}$ \\
\hline $\begin{array}{c}\mathrm{IMC} \\
\left(\mathrm{kg} / \mathrm{m}^{2}\right)^{* *}\end{array}$ & 397 & $\begin{array}{c}28.77 \\
(28.01-29.54)\end{array}$ & 4181 & $\begin{array}{c}27.54 \\
(27.30-27.78)\end{array}$ \\
\hline
\end{tabular}

+ Consumo de 100 cigarros en el último año, ** Consumo de alcohol en el último año. CRP=Proteína c reactiva, sTFR= Receptores solubles de transferrina, IMC= Índice de masa corporal, IC= Intervalo de confianza. $* \mathrm{p}<0.05$

$* * p<0.01$

$* * * \mathrm{p}<0.001$

2.- Asociación entre diabetes, ferritina y CRP.

En el modelo de regresión logística multivariada, la posibilidad de tener DM2 se asoció con las concentraciones séricas de ferritina. Los sujetos en el quintil 5 de ferritina tuvieron 2.11 veces más posibilidades de presentar DM2 en comparación con los sujetos del quintil 1, ajustando por IMC, sexo y edad. Al ajustar por CRP, tabaquismo y consumo de alcohol la atenuación de la asociación fue poca, obteniendo que los sujetos en el quintil 5 de ferritina tuvieron 1.97 veces más posibilidades de presentar DM2 (O.R. 1.97(1.11-3.52)) (Ver tabla 2). El efecto que se observa respecto a los quintiles refiere un umbral, no habiendo asociación en el quintil 2 y 3 con respecto al quintil1, pero al pasar quintil 4 el OR se duplica, existiendo una asociación, la cual se mantiene en el quintil 5, sugiriendo que existe un valor crítico (Ver figura 1).

De la misma manera la probabilidad de ser diabético se asoció con las concentraciones de CRP. Es decir, por cada $\mathrm{mg} / \mathrm{L}$ de CRP la probabilidad de ser diabético aumenta 0.01 [OR.1.01(1.0-1.07)]. Así mismo, por cada unidad más de IMC la posibilidad de ser diabético aumenta 0.03 veces [OR.1.03(1.01.03)]; Las concentraciones de sTFR, el sexo y el consumo de alcohol no mostraron ningún efecto sobre la posibilidad de presentar DM2, por lo tanto, no se muestra asociación (Ver tabla 3 ).

Tabla 2. Análisis de regresión logística de la asociación de ferritina y

\begin{tabular}{|c|c|c|c|c|c|}
\hline $\begin{array}{c}\text { Quintiles de } \\
\text { Ferritina }\end{array}$ & $\begin{array}{c}\text { Media de } \\
\text { ferritina } \\
\mathrm{ng} / \mathrm{ml}\end{array}$ & $\begin{array}{l}\text { \% sujetos } \\
\text { con DT2 }\end{array}$ & $\begin{array}{c}\text { OR (IC 95\%) } \\
\text { Modelo } 1 \\
\end{array}$ & $\begin{array}{c}\text { OR (IC95\%) } \\
\text { Modelo } 2 \\
\end{array}$ & $\begin{array}{c}\text { OR (IC 95\%) } \\
\text { Modelo } 3 \\
\end{array}$ \\
\hline uintil 1 & 9.58 & 5.7 & 1 & 1 & 1 \\
\hline Quintil 2 & 33.05 & 7.3 & $\begin{array}{c}1.14 \\
(.63-2-06)\end{array}$ & $\begin{array}{c}1.16 \\
(.63-2.08)\end{array}$ & $\begin{array}{c}1.16 \\
(.65-2.07)\end{array}$ \\
\hline Quintil 3 & 65.94 & 8.3 & $\begin{array}{c}1.16 \\
(.64-2.11)\end{array}$ & $\begin{array}{c}1.16 \\
(.64-2.11)\end{array}$ & $\begin{array}{c}1.16 \\
(.63-2.12)\end{array}$ \\
\hline Quintil 4 & 112.9 & 14.4 & $\begin{array}{c}2.05 * * \\
(1.07-3.92)\end{array}$ & $\begin{array}{c}1.99 * * \\
(1.05-3.75)\end{array}$ & $\begin{array}{c}1.96 * * \\
(1.04-3.71)\end{array}$ \\
\hline Quintil 5 & 247.43 & 15.7 & $\begin{array}{c}2.11^{* * *} \\
(1.18-3.78) \\
\end{array}$ & $\begin{array}{c}2.02 * * * \\
(1.13-3.6) \\
\end{array}$ & $\begin{array}{c}1.97^{* *} \\
(1.11-3.52) \\
\end{array}$ \\
\hline \multicolumn{6}{|c|}{$\begin{array}{l}\text { Modelo } 1 \text { (ajustado por edad, sexo e índice de masa corporal). Modelo } 2 \text { (ajustado } \\
\text { por variables modelo } 1+\text { consumo de alcohol y tabaquismo). Modelos } 3 \text { (ajustado } \\
\text { por variables modelo } 2+\text { proteína c reactiva). } O R=\text { Razón de momios. IC= Intervalos de } \\
\text { confianza. }\end{array}$} \\
\hline $\begin{array}{l}* n=4,578 \\
* * p<0.05 \\
* * * p=0.01\end{array}$ & & & & & \\
\hline
\end{tabular}

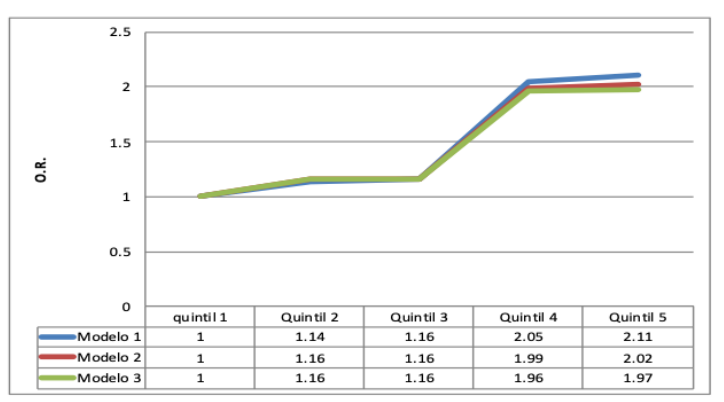

Figura 1. O.R. de diabetes según quintiles de ferritina. Modelo de regresión logística multivariada, asociación entre quintiles de ferritina y la posibilidad de presentar diabetes.

Modelo 2 (ajustado por variables modelo $1+$ consumo de alcohol y tabaquismo). Modelos 3 (ajustado por variables modelo $2+$ proteína c reactiva). 
Diabetes tipo 2, inflamación, proteína c-reactiva, ferritina.

Tabla 3. Regresión logística multivariada teniendo como variable dependiente la posibilidad de tener Diabetes tipo 2*

\begin{tabular}{|c|c|}
\hline Variable & OR (IC 95\%) \\
\hline $\mathrm{CRP} * *$ & $1.01(1.0-1.07)$ \\
\hline IMC** & $1.03(1.0-1.03)$ \\
\hline sTFR & $1.02(0.91-1.14)$ \\
\hline \multicolumn{2}{|l|}{ Edad (años) } \\
\hline $20-40$ & 1 \\
\hline $41-60 * * *$ & $5.82(3.54-9.55)$ \\
\hline $61-90 * * *$ & $10.58(6.27-17.84)$ \\
\hline \multicolumn{2}{|l|}{ Sexo } \\
\hline Mujer & 1 \\
\hline Hombre & $0.65(0.40-1.06)$ \\
\hline \multicolumn{2}{|l|}{ Tabaquismo $^{1}$} \\
\hline Si & $1.67(.99-2.83)$ \\
\hline No & $1.08(0.63-1.85)$ \\
\hline Nunca & 1 \\
\hline \multicolumn{2}{|c|}{ Consumo de alcohol ${ }^{2}$} \\
\hline Nunca & 1 \\
\hline Diario & $1.71(0.62-4.96)$ \\
\hline Semanal & $1.09(0.55-2.13)$ \\
\hline Mensual & $0.75(0.23-2.48)$ \\
\hline Ocasional & $1.2(0.73-1.97)$ \\
\hline \multicolumn{2}{|c|}{$\begin{array}{l}\text { 1. Consumo de } 100 \text { cigarros en el último año, } 2 . \\
\text { Consumo de alcohol en el último año, } \\
\text { CRP=Proteína c reactiva, sTFR= Receptores } \\
\text { solubles de transferrina, IMC= Índice de masa } \\
\text { corporal, IC= Intervalo de confianza, OR= Razón } \\
\text { demomios. }\end{array}$} \\
\hline \multicolumn{2}{|l|}{$*_{n}=4,578$} \\
\hline \multicolumn{2}{|l|}{$* * \mathrm{p}<0.05$} \\
\hline$* * * \mathrm{p}=0.001$ & \\
\hline
\end{tabular}

3.-Asociaciones entre ferritina, Proteína $\mathrm{C}$ reactiva y sTfR.

En la regresión lineal múltiple se obtuvo un valor R2 de 0.23 , lo cual explica solo una parte de la variabilidad de la ferritina. En este sentido, las concentraciones de sTFR $(\beta-0.91, \quad p<0.001)$ aportaron la mayor contribución a la variabilidad a las concentraciones de ferritina, seguidas por la variable sexo [(hombres) $(\beta 0.78, \quad \mathrm{p}<0.0001)]$, posteriormente las edades de 61 a 90 años $(\beta 0.45$, $\mathrm{p}<0.0001)$ y de 41 a 60 años $(\beta 0.29, \mathrm{p}<0.0001)$; y por último la diabetes $(\beta 0.24 \mathrm{p}<0.007)$ y la $\mathrm{CRP}(\beta 0.11$, $\mathrm{p}<0.0001)$ (Ver tabla 4).
Tabla 4. Modelo de regresión lineal múltiple teniendo como variable dependiente las concentraciones de ferritina. ${ }^{\text {\& }}$

\begin{tabular}{|c|c|}
\hline Variable & $\begin{array}{c}\text { Coeficiente } \beta \text { estandarizado } \\
\text { ( IC 95\%) }\end{array}$ \\
\hline Log proteína $\mathrm{C}$ reactiva** & $0.11(0.07,0.15)$ \\
\hline IMC & $0.007(-0.003,0.01)$ \\
\hline Log STFR** & $-0.91(-1.07,-0.75)$ \\
\hline \multicolumn{2}{|l|}{ Edad (años) } \\
\hline $20-40$ & 1 \\
\hline $41-60 * *$ & $0.29(0.17,0.40)$ \\
\hline $61-90^{* *}$ & $0.41(0.29,0.61)$ \\
\hline \multicolumn{2}{|l|}{ Tabaquismo+ } \\
\hline $\mathrm{Si}$ & $0.09(-0.03,0.22)$ \\
\hline No & $-0.04(-0.22,0.12)$ \\
\hline Nunca & 1 \\
\hline \multicolumn{2}{|l|}{ Consumo de alcohol } \\
\hline Diario & $0.15(-0.13,0.43)$ \\
\hline Semanal & $0.04(-0.11,0.20)$ \\
\hline Mensual & $0.10(-0.14,0.36)$ \\
\hline Ocasional & $-0.01(-0.14,0.11)$ \\
\hline Nunca & 1 \\
\hline Diabetes* & $0.24(0.06,0.42)$ \\
\hline \multicolumn{2}{|l|}{ Sexo } \\
\hline Mujer & 1 \\
\hline Hombre** & $0.78(0.66,0.90)$ \\
\hline
\end{tabular}

+ Consumo de por lo menos 100 cigarros en el úl timo año, Log= Logari tmo, IMC= İndice de masa corporal, STFR= Receptores solubles de transferrina.

$\&_{n=4,578, R^{2}=0.23}$

$* p<0.01$

$* * p=0.0001$

4.- Asociación entre ferritina y hemoglobina glucosilada

Considerando el análisis sobre la asociación de estas dos variables, ferritina como variable dependiente y HbA1c como variable independiente. Se encontró en esta muestra que la media de edad fue de 56.5 años, IMC de $28.89 \mathrm{~kg} / \mathrm{m} 2$, la distribución de mujeres fue de $59.5 \%$ y de hombres $40.5 \%$. El $6.6 \%$ tuvieron un buen control glucémico ( $\mathrm{HbA} 1 \mathrm{c}<7 \%)$, mientras que el $93.4 \%$ tuvieron mal control glucémico (HbA1c $\geq 7 \%$ ), la media de HbAlc fue de $10.2 \%$. Las medias geométricas de las variables bioquímicas fueron: ferritina $90.7 \mathrm{ng} / \mathrm{mL}$, sTFR $2.50 \mathrm{mg} / \mathrm{L}$, hierro sérico $90.46 \mu \mathrm{g} / \mathrm{dL}$ y CRP $3.22 \mathrm{mg} / \mathrm{L}$. 
Las concentraciones de $\mathrm{HbA} 1 \mathrm{c}$ no se asociaron significativamente con las concentraciones de ferritina en la regresión lineal [ Coeficiente $\beta-0.022$, IC $95 \%(-.05, .01)] \mathrm{p}=0.31$.

\section{Discusión.}

En este estudio se confirmó que las concentraciones medias de ferritina sérica son mayores en sujetos con DM2 en comparación con sujetos sin diabetes, como lo han encontrado varios estudios hechos en poblaciones de distintos orígenes étnicos (Akter et al.,2017; Canturk et al., 2003; Ford y Cogswell, 1999; Forouhi et al., 2007; Fumeron, P'eanc, Pharmd y Balkau., 2006; Jiang,2004; Ma, 2018; Salonen et al., 1998; Sharifi y Sazandeh, 2004).

Salonen et al., (1998) en su estudio de casos y controles anidados en una cohorte realizada en Finlandia con un seguimiento de 4 años, donde su objetivo fue evaluar la acumulación de hierro en el cuerpo como predictor de DM2, encontró que al finalizar el estudio los hombres con concentraciones elevadas de ferritina tuvieron un O.R.2.4 (I.C. 95\% 1.03- 5.5, $\mathrm{p}=0.04$ ), es decir $140 \%$ más posibilidades de tener DM2 en comparación con los hombres con bajos niveles de hierro. Ford et al., (1999) hicieron un estudio transversal en Estados Unidos, para examinar la relación entre la ferritina sérica y DM2, la población de estudio fue 9486 adultos de $\geq 20$ años evaluados en la National Health and Nutrition Examination Survey (NHANES; 1988-1994); identificándose 4 categorías para DM2, utilizando los criterios de la Organización Mundial de la Salud (OMS): sin DM2, intolerancia a la glucosa, nuevo diagnóstico de DM2 y diagnóstico previo. Para el quintil más alto de ferritina el O.R. para nuevo diagnóstico de DM2 fue de 4.94 (3.05-8.01) para hombres y 3.61 (2.01-6.48) para mujeres después de ajustar por edad, sexo, etnicidad, educación, IMC, consumo de alcohol y CRP.

Asimismo, se ha demostrado que las concentraciones de ferritina sérica se asocian a otros indicadores del estado nutricio de hierro, como las concentraciones de sTFR y de hierro sérico, demostrando que son las variables con mayor contribución a la variabilidad de las concentraciones de ferritina (sTFR: $\beta-0.91$ ). No se demostró que la variabilidad de las concentraciones de ferritina se asociase a los niveles de HbAlc, a diferencia del estudio de Son (2019), donde encontró que los niveles de ferritina sérica de los pacientes con DM2 aumentaron significativamente al aumentar niveles de HbA1c (p $<0,01)$ y una fuerte correlación positiva entre los niveles de ferritina sérica y HbA1c y niveles de glucosa en sangre en ayunas (FBG) $(p<0.01)$.

Derivado a que la CRP y la ferritina son reactantes de fase aguda (Horvat, 2020) ambos se aumentan en presencia de un proceso inflamatorio; se sugiere que la asociación que se demuestra en este estudio entre un indicador de inflamación aguda y crónica como es la CRP y la ferritina apunta que otra parte de la variabilidad de las concentraciones de ferritina sérica en diabéticos se debe al proceso inflamatorio crónico de baja intensidad que ocurre en la diabetes y en la obesidad (considerada en este estudio con el IMC) ( ; O'Rourker, 2013; Van den Oever et al., 2010). Diversos estudios epidemiológicos soportan el papel de la inflamación en la patogénesis de la DM2, se ha utilizado la CRP como indicador de inflamación y ha sido un predictor independiente de DM2 en diferentes poblaciones (Denghan, 2007; Doi, et al., 2005; Hu, Meigs, Li, Rifai y Manson, 2004; Pradham et al., 2001). Los mecanismos por los cuales las altas concentraciones de ferritina pudieran asociarse con la presencia de DM2 aún no son claros, se ha postulado que un mayor depósito de hierro en el hígado puede causar resistencia a la insulina, interfiriendo con su capacidad de suprimir la producción hepática de glucosa. El enlace entre niveles altos de ferritina y presencia de inflamación para asociarse con la DM2 podría estar determinada al menos en parte, por la alta capacidad del hierro para funcionar como un agente prooxidante sugiere que puede generar radicales libres aumentando el estrés oxidativo y con ello un mayor riesgo para desarrollar DM2 (Forouhi et al.,2007). Es importante señalar que este estudio muestra resultados convincentes según lo referido por otros autores que han evidenciado una asociación positiva entre ferritina y DM2; sin embargo, la evidencia es limitada a poblaciones europeas, asiáticas, afroamericanas y americanas, en México fue el primer estudio en mostrar estos datos provenientes de una encuesta nacional. Dentro de las limitaciones de este estudio están la falta de marcadores que identifiquen a sujetos con Hemocromatosis hereditaria, cuyo diagnóstico se basa en la existencia de concentraciones extremadamente altas de ferritina y de saturación de transferrina, además de excesivos 

ferritina.

depósitos de hierro en una biopsia hepática o bien la identificación de polimorfismos del gen de HFE (Pietrangelo, 2004a, 2010b). Con el propósito de minimizar esta posibilidad se excluyó del análisis a los sujetos con concentraciones de ferritina $\geq 1000 \mathrm{ng} / \mathrm{ml}$. En cuanto a la submuestra analizada para determinar la asociación entre ferritina y HbA1c el poder estadístico fue muy bajo del $25 \%$, es muy probable que esto se deba al tamaño de muestra tan pequeño de sujetos con determinaciones simultaneas de HbA1c y ferritina. Lo anterior sugiere que falta de asociación podría deberse al bajo poder estadístico y estar cometiendo un error tipo II; además dado el diseño de este estudio es difícil interpretar las asociaciones, así como su plausibilidad biológica, para tal caso se deben realizar estudios longitudinales que puedan observar los efectos.

\section{Conclusiones}

Este estudio muestra, que en sujetos mexicanos con DM2 las concentraciones de ferritina sérica son mayores que en sujetos sin diabetes y que la mayor parte de su variabilidad está explicada por la variación de los indicadores del estatus de hierro y de inflamación (CRP). No se pudo establecer que la HbA1c tenga impacto sobre las concentraciones de ferritina. Es importante establecer que es necesario realizar futuros estudios que ayuden a entender los mecanismos implicados en estos eventos, como estudios longitudinales que contemplen más variables para tratar de explicar sus efectos sobre las concentraciones de ferritina, además ensayos clínicos que desarrollen intervenciones que permitan establecer direccionalidad y posibles efectos de variables sobre las concentraciones de ferritina.

\section{Bibliografía}

Akter, S., Nanri, A., Kuwahara, K., Matsushita, Y., Nakagawa, T., \& Konishi, M. et al. (2017). Circulating ferritin concentrations and risk of type 2 diabetes in Japanese individuals. Journal of Diabetes Investigation, 8(4), 462-470. doi: $10.1111 /$ jdi.12617

Canturk, Z., Çetinarslan, B., Tarkun, İ., \& Zafer Canturk, N. (2003). Serum Ferritin Levels in Poorly- and Well-Controlled Diabetes Mellitus. Endocrine Research, 29(3), 299-306. doi: 10.1081/erc-120025037
Carrillo E., Peña P., Zepeda M. (2015). Ferritina y síndrome hiperferritinémico. Su impacto en el enfermo grave; conceptos actuales. Med Crit. 2015;29(3),157-166.

Dehghan A., Hoek M., Sijbrands E., Stijnen T., Witteman J., \& Hofman A. (2007). Risk of type 2 diabetes attributable to c-reactive protein and other risk factors. Diabetes Care, 30:2695-2699.

Doi Y., Kiyohara Y., Kubo M., Ninomiya T., Wakugwa Y., \& Yonemoto K. (2005). Elevated c-reactive protein is a predictor of the development of diabetes in a general japanese population. Diabetes Care, 28:2497-2500. doi: 10.2337/diacare.28.10.2497

Eshed, I., Elis, A., \& Lishner, M. (2001). Plasma Ferritin and Type 2 Diabetes Mellitus: A Critical Review. Endocrine Research, 27(1-2), 91-97. doi: 10.1081/erc-100107172

Fernandez-Real, J., Lopez-Bermejo, A., \& Ricart, W. (2002). Crosstalk Between Iron Metabolism and Diabetes. Diabetes, 51(8), 2348-2354. doi: 10.2337/diabetes.51.8.2348

Ford, E., \& Cogswell, M. (1999). Diabetes and serum ferritin concentration among U.S. adults. Diabetes Care, 22(12), 1978-1983. doi: 10.2337/diacare.22.12.1978

Forouhi, N., Harding, A., Allison, M., Sandhu, M., Welch, A., \& Luben, R. et al. (2007). Elevated serum ferritin levels predict new-onset type 2 diabetes: results from the EPIC-Norfolk prospective study. Diabetologia, 50(5), 949-956. doi: 10.1007/s00125-007-0604-5

Fumeron F., P'ean F., Pharmd F., \& Balkau B. (2006). Ferritin and Transferrin Are Both Predictive of the Onset of Hyperglycemia in Men and Women Over 3 Years. Diabetes Care, 29:2090-2094.

Hu F., Meigs J., Li T., Rifai N., \& Manson J. (2004). Inflammatory markers and risk of developing type 2 diabetes in women. Diabetes, 53:693-700. doi: $10.2337 /$ diabetes.53.3.693 
Jehn, M. L., Guallar, E., Clark, J. M., Couper, D., Duncan, B. B., Ballantyne, C. M., et al. (2005). A prospective study of plasma ferritin level and incident diabetes: the Atherosclerosis Risk in Communities (ARIC) Study. Am. J. Epidemiol, 165(9): 1047-54.

Jiang, R. (2004). Body Iron Stores in Relation to Risk of Type 2 Diabetes in Apparently Healthy Women. JAMA, 291(6), 711. 10.1001/jama.291.6.711

Ma, H., Lin, H., Hu, Y., Li, X., He, W., \& Jin, X. et al. (2018). Serum ferritin levels are associated with insulin resistance in Chinese men and postmenopausal women: The Shanghai Changfeng study. British Journal of Nutrition, 120(8), 863871. doi: $10.1017 / \mathrm{s} 0007114518002167$

O'Rourke, R. (2009). Molecular Mechanisms of Obesity and Diabetes: At the Intersection of Weight Regulation, Inflammation, and Glucose Homeostasis. World Journal of Surgery, 33(10), 2007-2013. doi: 10.1007/s00268-009-0067-6

Pickup, J. (2004). Inflammation and Activated Innate Immunity in the Pathogenesis of Type 2 Diabetes. Diabetes Care, 27(3), 813-823. doi: 10.2337/diacare.27.3.813

Pietrangelo A. (2004). Hereditary Hemochromatosis- A new look at an old disease. $N$ Engl J Med, 350:2383-2397. doi: 10.1056/NEJMra031573

Pietrangelo A. (2010). Hereditay Hemochromatosis: Pathogenesis, Diagnosis, and treatment. Gastroenterology, 139(2):393-408,408.e12. doi:10.1053/j.gastro.2010.06.013.

Pradham A., Manson J., Rifai N., Buring J., \& Ridker P. (2001). C-reactive protein, Interleukin 6 and risk developing type 2 diabetes mellitus. JAMA, 286:327-334. doi: 10.1001/jama.286.3.327

Rajpathak, S. N., Wylie-Rosett, J., Gunter, M. J., Negassa, A., Kabat, G. C., Rohan, T. E., et al. (2009). Biomarkers of body iron stores and risk of developing type 2 diabetes. Diab Obes. Metab, 11(5), 472-9.
Rojas-Martínez, R., Basto-Abreu, A., AguilarSalinas, C., Zárate-Rojas, E., Villalpando, S., \& Barrientos-Gutiérrez, T. (2018). Prevalencia de diabetes por diagnóstico médico previo en México. Salud Pública de México, 60(3, mayjun), 224. doi: 10.21149/8566

Salonen J., Tuomainen T., Nyyssönen K., Lakka H., \& Punnonen K. (1998). Relation between iron stores and non-insulin dependent diabetes in men: case-control study. BMJ, 317(7160): 727730 .

Shamah-Levy T., Villalpando-Hernández S., \& Rivera-Dommarco JA. (2007). Resultados de Nutrición de la ENSANUT 2006. Cuernavaca, México: Instituto Nacional de Salud Pública.

Shamah- Levy T., Villalpando- Hernández S., \&Rivera-Dommarco J. (2006). Manual de Procedimientos para Proyectos de Nutrición. Cuernavaca, México. Instituto Nacional de Salud Pública.

Sharifi F., \& Sazandeh S. (2004). Serum ferritin in type 2 diabetes mellitus and its relationship with HbA1c. Acta Med Iranica, 42(2): 142-145.

Son N. (2019). Influence of ferritin levels and inflammatory markers on HbA1c in the Type 2 Diabetes mellitus patients. Pak J Med Sci, 35(4), 1030-1035. doi: https://doi.org/10.12669/pjms.35.4.1003

Sun, L., Zong, G., Pan, A., Ye, X., Li, H., Yu, Z., et al. (2013). Elevated plasma ferritin is associated with increased incidence of type 2 diabetes in middle-aged and elderly Chinese adults. J. Nutr, 143(9), 1459-65.

Swaminathan, S., Fonseca, V., Alam, M., \& Shah, S. (2007). The Role of Iron in Diabetes and Its Complications. Diabetes Care, 30(7), 19261933. doi: $10.2337 / \mathrm{dc} 06-2625$

Twetz NW (1999). Textbook of Clinical Chemistry (pp. 794-795). Philadelphia: Saunders. 
Diabetes tipo 2, inflamación, proteína c-reactiva, ferritina.

Van den Oever, I., Raterman, H., Nurmohamed, M., \& Simsek, S. (2010). Endothelial Dysfunction, Inflammation, and Apoptosis in Diabetes Mellitus. Mediators of Inflammation, 2010, 1-15. doi: $10.1155 / 2010 / 792393$

Villalpando S., Rojas R., Shaman-Levy T., Avila M., Gaona B., De la Cruz V., Rebollar R., \& Hernandez L. (2010). Prevalence and distribution of type 2 Diabetes mellitus in Mexican adult population. A probabilistic survey. Salud Publ Mex, 52(1): S19-S26.

World Health Organization. Obesity: Preventing and managing the Global Epidemic. WHO technical Report Series 2000;894. [consultado 23/01/20]. Disponible

en: https://www.who.int/nutrition/publications/obesi ty/WHO_TRS_894/en/ 